\title{
Identification of a multi-dimensional reaction coordinate for crystal nucleation in $\mathrm{Ni}_{3} \mathrm{Al}$
}

Cite as: J. Chem. Phys. 152, 224504 (2020); https://doi.org/10.1063/5.0010074

Submitted: 07 April 2020 . Accepted: 21 May 2020. Published Online: 12 June 2020

(iD) Yanyan Liang (梁燕燕), (D) Grisell Díaz Leines, Ralf Drautz, and (D) Jutta Rogal

\section{ARTICLES YOU MAY BE INTERESTED IN}

Role of pre-ordered liquid in the selection mechanism of crystal polymorphs during nucleation

The Journal of Chemical Physics 153, 104508 (2020); https://doi.org/10.1063/5.0017575

Classical nucleation theory predicts the shape of the nucleus in homogeneous solidification The Journal of Chemical Physics 152, 044103 (2020); https://doi.org/10.1063/1.5134461

Atomistic insight into the non-classical nucleation mechanism during solidification in $\mathrm{Ni}$

The Journal of Chemical Physics 146, 154702 (2017); https://doi.org/10.1063/1.4980082

\section{Challenge us.}

What are your needs for periodic signal detection?

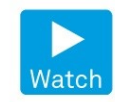

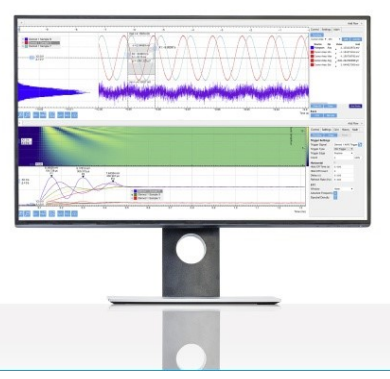

Zurich Instruments

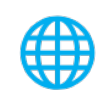

View Online 


\title{
Identification of a multi-dimensional reaction coordinate for crystal nucleation in $\mathrm{Ni}_{3} \mathrm{Al}$
}

\author{
Cite as: J. Chem. Phys. 152, 224504 (2020); doi: 10.1063/5.0010074 \\ Submitted: 7 April 2020 - Accepted: 21 May 2020 • \\ Published Online: 12 June 2020
}

Yanyan Liang (梁燕燕), (D) Grisell Díaz Leines, (D) Ralf Drautz, and Jutta Rogal ${ }^{\text {b) }}$

\author{
AFFILIATIONS \\ Interdisciplinary Centre for Advanced Materials Simulation, Ruhr-Universität Bochum, 44780 Bochum, Germany
}

a) Electronic mail: yanyan.liang@rub.de

b) Author to whom correspondence should be addressed: jutta.rogal@rub.de

\begin{abstract}
Nucleation during solidification in multi-component alloys is a complex process that comprises competition between different crystalline phases as well as chemical composition and ordering. Here, we combine transition interface sampling with an extensive committor analysis to investigate the atomistic mechanisms during the initial stages of nucleation in $\mathrm{Ni}_{3} \mathrm{Al}$. The formation and growth of crystalline clusters from the melt are strongly influenced by the interplay between three descriptors: the size, crystallinity, and chemical short-range order of the emerging nuclei. We demonstrate that it is essential to include all three features in a multi-dimensional reaction coordinate to correctly describe the nucleation mechanism, where, in particular, the chemical short-range order plays a crucial role in the stability of small clusters. The necessity of identifying multi-dimensional reaction coordinates is expected to be of key importance for the atomistic characterization of nucleation processes in complex, multi-component systems.
\end{abstract}

Published under license by AIP Publishing. https://doi.org/10.1063/5.0010074

\section{INTRODUCTION}

Fundamental knowledge of crystal nucleation in multicomponent systems is essential for the controlled synthesis of materials with targeted properties. Despite this, insight into the atomistic mechanisms of nucleation remains elusive as even simple model systems exhibit complex transitions that involve several steps, polymorphic structures, and multiple order parameters. ${ }^{1,2}$ For multicomponent systems, the additional complexity that emerges from possible ordering tendencies of the chemical species poses a major challenge for both experiment and theory. Consequently, even less is known about the underlying mechanisms that govern the initial steps of crystallization in technological relevant alloys.

Characterizing the mechanism of a complex, activated process such as nucleation is intrinsically linked to identifying the degrees of freedom that represent a relevant reaction coordinate (RC). On the mesoscale, classical nucleation theory $(\mathrm{CNT})^{3,4}$ has been successfully applied to study nucleation. One of the key assumptions in CNT is that crystal nucleation is a one-dimensional process described entirely by a single RC, namely, the radius of a spherical nucleus or, more generally, the size of the growing cluster. However, several theoretical studies have shown that even for simple systems such as Lennard-Jones (LJ) liquids, hard spheres, and colloidal suspensions, a one-dimensional RC is not sufficient to correctly capture the nucleation mechanism. ${ }^{2,5-11}$ Indeed, crystal nucleation is inherently a multi-dimensional process that has been described as the ordering of multiple order parameters, ${ }^{2}$ where a trade-off between entropy and enthalpy ${ }^{12}$ characterizes the transition, and a decoupling of translational and orientational order can occur at different temperatures. $^{2}$ In binary or multi-component systems where changes in chemical order and composition as well as demixing can play a key role during nucleation and growth, the identification of meaningful, low-dimensional collective variables (CVs) that can serve as RCs is extremely challenging.

Theoretically, the committor ${ }^{13-15}$ can be considered as the optimal RC of an activated process. ${ }^{16}$ The committor provides a statistical measure of the progress of a transition between two states by measuring the probability for any given configuration to commit to the final state. Due to its statistical definition, the committor does not yield direct physical insight into the mechanism, yet it can be used to evaluate the quality of physically meaningful collective variables as RCs. Good CV candidates for the reaction coordinate should, for example, exhibit a strong correlation with the committor. For studies of crystal nucleation in single-component systems such 
as LJ systems, soft core colloids, and pure metals, ${ }^{6,17,18}$ an extensive committor analysis revealed that the size of the crystalline nucleus is not sufficient to model the RC, and additional variables such as the crystallinity or a cloud of pre-structured surface particles around a crystalline core improve the description of the mechanism.

Several theoretical studies have investigated crystal nucleation in two-component systems, including bimetallics of $\mathrm{Pd}-\mathrm{Ag}$, $\mathrm{Cu}-\mathrm{Ni}, \mathrm{Pd}-\mathrm{Ni}, \mathrm{Cu}-\mathrm{Pd},{ }^{19-22}$ and mixtures of colloidal particles. ${ }^{23-29}$ The bimetallic systems above have rather simple phase diagrams forming solid solution bulk phases without strong chemical ordering tendencies. Consequently, the main focus of these studies is on demixing and the enrichment of chemical species in the growing cluster. ${ }^{19-22}$ The initial crystal nucleation process can, however, be considerably different for chemically ordered phases compared to random solid solutions. Indeed, studies of crystal nucleation in colloidal mixtures showed that the nucleation mechanism is largely affected by the underlying complex phase diagram containing ordered compounds. Competing structures of $\mathrm{CsCl}$ ordered body-centered cubic (bcc) and disordered face-centered cubic (fcc) were found during nucleation, ${ }^{27-29}$ and structure-specific parameters were required to differentiate competing pathways that lead to different bulk phases. ${ }^{2}$

In this work, we investigate the mechanisms of crystal nucleation in binary $\mathrm{Ni}_{3} \mathrm{Al}$. Ni-Al alloys are of particular interest since they serve as a basis for high-performance materials that are key in many technological applications. ${ }^{30}$ In contrast to the bimetallic alloys mentioned previously, $\mathrm{Ni}-\mathrm{Al}$ exhibits a fairly complicated phase diagram with a number of chemically ordered structures. ${ }^{31-33}$ Specifically, $\mathrm{Ni}-\mathrm{Al}$ exhibits a single phase region of $\mathrm{Ni}_{3} \mathrm{Al}$ ranging from 73 at. $\%$ to 77 at. $\% \mathrm{Ni}$ in the equilibrium phase diagram ${ }^{32}$ where it forms an $\mathrm{L}_{2}$ ordered fcc structure that is thermodynamically stable up to the melting temperature. It was, however, shown in electromagnetic levitation experiments that during rapid solidification in Ni-rich alloys with $23.5-30.8$ at. \% Al, there is a strong competition between the formation of several chemically ordered and disordered fcc and bcc phases. ${ }^{34}$ However, it remains unclear how this competition between various chemically ordered and disordered phases influences the nucleation mechanism, at what stage of the nucleation process this becomes relevant, and how it is reflected in a set of CVs needed to characterize the mechanism. To address these open questions, we have combined transition interface sampling (TIS), ${ }^{35}$ for an enhanced sampling of the nucleation process in $\mathrm{Ni}_{3} \mathrm{Al}$, with a committor analysis ${ }^{13,36}$ to assess the quality of proposed CVs as reaction coordinates. We find that during crystal nucleation in $\mathrm{Ni}_{3} \mathrm{Al}$, there is indeed a competition between various crystalline structures, strongly indicating the existence of multiple reaction channels. As a consequence, the size of the growing cluster as a single $\mathrm{RC}$ results in an incomplete description of the nucleation mechanism in contrast to the nucleation in unary metals. ${ }^{37,38}$ In addition to the size, information concerning the crystallinity as well as the chemical short-range order (SRO) is required to differentiate potential nucleation pathways. In particular, the chemical SRO is discovered to be a crucial factor triggering continuous growth or shrinkage of solid clusters with the same size and crystallinity. The initial stage of crystal nucleation in $\mathrm{Ni}_{3} \mathrm{Al}$ exhibits an enormous complexity caused by the interplay between size, crystallinity, and short-range order. As a result, all of these aspects need to be included in the $\mathrm{RC}$ for an accurate representation of the nucleation process. We expect that, generally in complex systems with competing nucleation pathways, the assessment of multi-dimensional reaction coordinates is of fundamental importance.

\section{METHODS}

\section{A. Committor analysis}

An approach widely used to analyze reaction coordinates is based on the concept of the commitment probability or committor, $p_{B} \cdot{ }^{13-15}$ In a system with two metastable states, $A$ and $B$, the committor $p_{B}(\mathbf{r})$ is defined as the probability that a trajectory starting at a configuration $\mathbf{r}$ reaches state $B$ before $A$, calculated as an average over the Boltzmann distributed momenta at a given temperature. The committor provides a statistical measure of the progress of the reaction from the initial state $A$ to the final state $B$ and is often considered the perfect RC. ${ }^{16}$ By definition, $p_{B}(\mathbf{r})=0$ for configurations within state $A$, and $p_{B}(\mathbf{r})=1$ for configurations within $B$. Configurations with $p_{B}(\mathbf{r})=0.5$ mark the separatrix or transition state (TS). The committor does not, however, yield any direct physical insight into the mechanisms of reactive events, but it can be used to evaluate the quality of collective variables, $\mathbf{q}(\mathbf{r})=\left\{q_{1}(\mathbf{r}), \ldots, q_{n}(\mathbf{r})\right\}$, as reaction coordinates. Specifically, for a good reaction coordinate, the $\mathrm{CVs}$ should be strongly correlated with the committor. The quality of any given vector $\mathbf{q}(\mathbf{r})$ of $\mathrm{CV}$ values as a reaction coordinate can be assessed on the basis of the committor distribution ${ }^{13,36}$

$$
P\left(\left.p_{B}\right|_{\mathbf{q}}\right)=\frac{\left\langle\delta\left(p_{B}(\mathbf{r})-p_{B}\right) \delta(\mathbf{q}(\mathbf{r})-\mathbf{q})\right\rangle}{\langle\delta(\mathbf{q}(\mathbf{r})-\mathbf{q})\rangle} .
$$

Here, $\langle\cdots\rangle$ denotes the ensemble average, and $\delta(\mathbf{z})=\prod_{i=1}^{n} \delta\left(z_{i}\right)$ is the Dirac delta function. A set of meaningful CVs that properly characterize the mechanism of the transition between $A$ and $B$ will yield a committor distribution with a single, sharp peak for any value of $\mathbf{q}$. If, for example, $\mathbf{q}=\mathbf{q}^{*}$ coincides with the transition state, the committor distribution should be narrowly peaked around $p_{B}=0.5$. In contrast, a wide spread, multimodal committor distribution is a clear sign of a poor choice of CVs as reaction coordinates.

A first indication of the quality of collective variables is provided by the averaged committor ${ }^{39}$

$$
\bar{p}_{B}(\mathbf{q})=\frac{\left\langle p_{B}(\mathbf{r}) \delta(\mathbf{q}(\mathbf{r})-\mathbf{q})\right\rangle}{\langle\delta(\mathbf{q}(\mathbf{r})-\mathbf{q})\rangle},
$$

which is given by integrating over $p_{B}$ in Eq. (1). The averaged committor needs to monotonically increase along a set of relevant CVs and can be used to propose tentative transition state ensembles with $\bar{p}_{B}(\mathbf{q})=0.5$ that can then be further scrutinized by analyzing the committor distribution.

\section{B. Transition path sampling}

Due to the rare event nature of nucleation processes, an advanced simulation technique is required to study the transition between states $A$ and $B$, in this case, the liquid and the solid. Here, we employ transition interface sampling, ${ }^{35}$ a variant of transition path sampling, ${ }^{13,36}$ that utilizes a progress parameter $\lambda$ to define a set of interfaces as hypersurfaces between the two metastable states. 
For each interface, $\lambda_{i}$, an ensemble of dynamical trajectories is harvested by a Monte Carlo (MC) sampling in path space. The individual path ensembles for each interface can be combined into a complete path ensemble by reweighting the path probabilities. This reweighted path ensemble (RPE) ${ }^{40}$ comprises the unbiased dynamics of the system in the full phase space and allows for a direct calculation of various rare event properties. ${ }^{39}$ In particular, the averaged committor in Eq. (2) can be projected from the RPE onto any set of collective variables,

$$
\bar{p}_{B}(\mathbf{q})=\frac{\int \mathscr{D} \mathbf{x}^{L} \mathscr{P}\left[\mathbf{x}^{L}\right] \mathbb{1}_{B}\left(\mathbf{x}_{L}\right) \sum_{k=0}^{L} \delta\left(\mathbf{q}\left(\mathbf{x}_{k}\right)-\mathbf{q}\right)}{\int \mathscr{D} \mathbf{x}^{L} \mathscr{P}\left[\mathbf{x}^{L}\right] \sum_{k=0}^{L} \delta\left(\mathbf{q}\left(\mathbf{x}_{k}\right)-\mathbf{q}\right)},
$$

where $\mathscr{P}\left[\mathbf{x}^{L}\right]$ is the reweighted path ensemble, $\int \mathscr{D} \mathbf{x}^{L}$ denotes the integral over all phase space trajectories of all lengths $L, \mathbf{x}^{L}=\left\{\mathbf{x}_{0}\right.$, $\left.\ldots, \mathbf{x}_{L}\right\}$, and $\mathbb{1}_{B}\left(\mathbf{x}_{L}\right)$ is an indicator function that is one if the last slice of the trajectory, $\mathbf{x}_{L}$, is in state $B$ and zero otherwise. To a first approximation, the averaged committor projected from the RPE in Eq. (3) can be used to examine the correlation of various CVs with the committor and identify possible transition state ensembles with $\bar{p}_{B}\left(\mathbf{q}^{*}\right) \approx 0.5$. By combining the RPE with a maximum likelihood estimation, ${ }^{16}$ it is even possible to achieve a quantitative comparison of the quality of different CVs as reaction coordinates. ${ }^{18,41,42}$

\section{Collective variables for $\mathrm{Ni}_{3} \mathrm{Al}$}

To study the nucleation mechanism in $\mathrm{Ni}_{3} \mathrm{Al}$, we consider a set of 22 different collective variables that comprise parameters concerning the size, crystal structure, and chemical species of the growing solid cluster.

\section{Size of the largest solid cluster}

A commonly used $\mathrm{CV}$ that is often comparable to the radius of the growing nucleus in CNT is the number of particles in the largest solid cluster, $n_{s}$. Solid and liquid particles are distinguished based on the Steinhardt bond order parameters. ${ }^{43}$ We use two criteria to identify solid particles: the first is counting the number of solid bonds of each atom $i$ by evaluating the correlation with its neighbors $j,{ }^{44} s_{i j}=\sum_{m=-6}^{6} q_{6 m}(i) q_{6 m}^{*}(j)$, where $q_{6 m}$ are the complex vectors calculated from the spherical harmonics with $l=6 .{ }^{43}$ If $s_{i j}>0.5$, the connection between $i$ and $j$ is considered as a solid bond. The second criterion evaluates the average of the correlation over all neighbors $N_{b}(i)$ of atom $i,{ }^{45}\left\langle s_{i}\right\rangle=1 / N_{b}(i) \sum_{j=1}^{N_{b}(i)} s_{i j}$, which improves the identification of solid- and liquid-like particles at the solid-liquid interface. If the number of solid bonds is larger than 7 and $\left\langle s_{i}\right\rangle>0.6$, an atom is considered as solid. The number of atoms in the largest solid cluster, $n_{s}$, is then determined by a clustering algorithm.

Furthermore, we define the number of skin $n_{\text {sk }}$ and core $n_{c}$ atoms. Skin atoms are solid particles in the largest cluster that have at least one liquid neighbor. Core atoms are part of the largest solid cluster and do not have any liquid neighbors. Correspondingly, the sum of skin and core atoms yields the largest solid cluster size, that is, $n_{s}=n_{\mathrm{sk}}+n_{c}$.

\section{Crystal structure identification}

The local crystalline structure around each atom is determined by employing the averaged version of the Steinhardt bond order parameters ${ }^{46}$ with $l=4,6$, that is, $\bar{q}_{4}(i)$ and $\bar{q}_{6}(i)$. A reference map in the $\bar{q}_{4}-\bar{q}_{6}$ space $^{10,17,37}$ was calculated for $\mathrm{Ni}_{3} \mathrm{Al}$ including probability distributions for fcc, bcc, hexagonal closed-packed (hcp), and liquid structures (see Fig. S1 of the supplementary material). An atom $i$ is assigned to the structure with the highest probability in the reference map for the corresponding $\bar{q}_{4}(i)-\bar{q}_{6}(i)$ value. If all probabilities are less than $10^{-5}$, the particle is labeled undefined.

Based on the local structure identification, we define additional collective variables comprising the fraction of fcc, bcc, hcp, and undefined atoms in the largest solid cluster, $\frac{n_{s}^{\text {fcc }}}{n_{s}}, \frac{n_{s}^{\text {bcc }}}{n_{s}}, \frac{n_{s}^{\text {hcp }}}{n_{s}}$, and $\frac{n_{s}^{\text {un }}}{n_{s}}$, and in the core of the largest cluster, $\frac{n_{c}^{\text {fcc }}}{n_{c}}, \frac{n_{c}^{\text {bcc }}}{n_{c}}, \frac{n_{c}^{\text {hep }}}{n_{c}}$, and $\frac{n_{c}^{\text {un }}}{n_{c}}$, respectively.

\section{Global crystallinity within the solid cluster}

In addition to a local structure identification, we consider a global orientational order parameter, ${ }^{44,47}$ $Q_{6}^{\mathrm{cl}}=\left(\frac{4 \pi}{13} \sum_{m=-6}^{6}\left|\frac{\sum_{i=1}^{N} N_{b}(i) q_{6 m}(i)}{\sum_{i=1}^{N} N_{b}(i)}\right|^{2}\right)^{\frac{1}{2}}$, where the sum runs over all particles in the largest solid cluster. ${ }^{6}$ This $\mathrm{CV}$ measures the degree of crystallinity within a solid cluster: solid clusters that are more compact and well ordered show high values of $Q_{6}^{\mathrm{cl}}$ and vice versa. In $\mathrm{Ni}_{3} \mathrm{Al}$, perfect fcc bulk exhibits the highest crystallinity value of all structures with $Q_{6}^{\mathrm{cl}}=0.48$.

\section{Chemical composition and short-range order}

In binary systems, collective variables assessing the chemical composition and the chemical order are also of interest, in particular, with respect to phase separation and order-disorder transitions. To monitor deviations from the ideal 3:1 ratio of nickel and aluminum in $\mathrm{Ni}_{3} \mathrm{Al}$, we determine the relative amount of $\mathrm{Al}$ in the liquid $\frac{n_{\text {liq }}^{\mathrm{Al}}}{n_{\mathrm{liq}}}$, the largest solid cluster $\frac{n_{s}^{\mathrm{Al}}}{n_{s}}$, the skin atoms $\frac{n_{\mathrm{sk}}^{\mathrm{Al}}}{n_{\mathrm{sk}}}$, and the core atoms $\frac{n_{c}^{\mathrm{Al}}}{n_{c}}$, respectively. Likewise, the fraction of $\mathrm{Al}$ for specific crystalline phases in the largest solid cluster is evaluated with $\frac{n_{s}^{\text {fcc-Al }}}{n_{s}^{\text {fcc }}}, \frac{n_{s}^{\text {bcc-Al }}}{n_{s}^{\text {bcc }}}, \frac{n_{s}^{\text {hcp-Al }}}{n_{s}^{\text {hcp }}}$, and $\frac{n_{s}^{\text {un-Al }}}{n_{s}^{\text {un }}}$.

The chemical order of $\mathrm{Ni}$ and $\mathrm{Al}$ is determined by a short-range order parameter ${ }^{48}$ that was suggested for measuring SRO in nanostructures, such as small clusters. The SRO parameter is a normalized pair-correlation function with spin-like variable $S_{i}$ for the site occupancy, that is, $S_{i}=1$ and -1 for atomic species A and B, respectively. For a binary alloy, the SRO parameter of site $i$ for the $n$th neighbor shell is defined as

$$
\mathrm{SRO}_{n}=\frac{\left\langle S_{i} S_{i+n}\right\rangle-\left\langle S_{i}\right\rangle^{2}}{1-\left\langle S_{i}\right\rangle^{2}}
$$

where $\left\langle S_{i}\right\rangle=2 x-1$ and $x$ is the overall fraction of the chemical element occupying site $i$, i.e., $x_{\mathrm{Ni}}=0.75$ and $x_{\mathrm{Al}}=0.25$ for $\mathrm{Ni}_{3} \mathrm{Al}$. If the lattice sites are randomly occupied by $\mathrm{Ni}$ and $\mathrm{Al}$, the correlation parameter is $\mathrm{SRO}_{n}=0$. For the chemically ordered $\mathrm{L}_{2}$ phase, $\mathrm{SRO}_{1}$ $=-1 / 3$ for the first, and $\mathrm{SRO}_{2}=1$ for the second neighbor shell. Since $\mathrm{Ll}_{2}$ is the most stable phase in $\mathrm{Ni}_{3} \mathrm{Al}^{31,32}$ we use these SRO parameter values as reference to quantify the chemical order of crystalline embryos that emerge within the melt. 


\section{Computational details}

All simulations were performed in a cubic box with 6912 atoms, corresponding to a $(12 \times 12 \times 12) \mathrm{fcc}$ supercell, and a fixed composition of 75 at. \% Ni and 25 at. \% Al. The interatomic interactions were modeled with an embedded atom method (EAM) potential for $\mathrm{Ni}-\mathrm{Al}^{49}$ that was shown to capture the stability of various stable and metastable phases across the phase diagram. The EAM potential gives a melting temperature for $\mathrm{L}_{2}$ ordered $\mathrm{Ni}_{3} \mathrm{Al}$ of approximately $T_{m}=1678 \mathrm{~K},{ }^{49}$ which agrees well with the experimental values of $T_{m}=1645 \mathrm{~K}^{32}$ Dynamical trajectories were created by molecular dynamics (MD) using the simulation package Large-scale Atomic/Molecular Massively Parallel Simulator (LAMMPS) ${ }^{50}$ in the isothermal-isobaric (NPT) ensemble with a Nosé-Hoover thermostat and barostat. The damping time regulating the temperature and pressure was set to 0.05 ps and 0.5 ps, respectively. Only the volume of the simulation box was allowed to change, while the shape was kept cubic. The pressure and temperature were $P=0$ bar and $T=1342 \mathrm{~K}$, which corresponds to an undercooling of $20 \%$ with respect to the melting temperature of $\mathrm{Ni}_{3} \mathrm{Al}$ given by the $\mathrm{Ni}-\mathrm{Al}$ EAM. $^{49}$ The integration time step was set to $\Delta t=1 \mathrm{fs}$, and threedimensional periodic boundary conditions were applied in all MD simulations.

For the TIS simulations, we adopted a python wrapper ${ }^{51}$ combined with LAMMPS to perform the MC sampling of the MD trajectories. As progress parameter $\lambda$, the size of the largest solid cluster $n_{s}$ was used. In total, there are 21 interfaces with $\lambda=\{15,25,38,50,63$, $80,100,125,140,170,200,230,260,300,320,330,350,400,450,475$, $500\}$. The positions of the interfaces are chosen such that there is at least $10 \%$ overlap in the crossing histograms between neighboring interfaces. The first interface $\lambda_{0}=15$ marks the boundary of the liquid state and the last one $\lambda_{20}=500$ of the solid state. For cluster sizes $n_{s} \geq 500$, the system is fully committed to the solid state and complete solidification occurs. The path ensemble was harvested with replica exchange TIS (RETIS) ${ }^{52,53}$ with $45 \%$ shooting moves, $45 \%$ exchange moves, and $10 \%$ exchange between the forward and backward ensembles. For each interface, $1800 \mathrm{MC}$ moves were performed where trajectories were collected after five decorrelation steps, generating 360 paths in each interface ensemble. All atomic positions and velocities were recorded at 0.2 ps time intervals.

To compute the committor distribution $P\left(\left.p_{B}\right|_{\mathbf{q}}\right)$ in Eq. (1), a minimum number of 50 configurations with a given value of $\mathbf{q}$ was randomly chosen from the path ensemble. For each configuration, $100 \mathrm{MD}$ simulations were initiated with Maxwell-Boltzmann distributed velocity at $T=1342 \mathrm{~K}$, and it was monitored if the trajectory entered the solid $\left(n_{s} \geq 500\right)$ or liquid $\left(n_{s} \leq 15\right)$ state first, yielding the committor $p_{B}(\mathbf{q})$. All $p_{B}(\mathbf{q})$ values are converged to within \pm 0.05 .

\section{RESULTS}

\section{A. Multiple reaction channels during nucleation}

An important step in analyzing the mechanism of nucleation in $\mathrm{Ni}_{3} \mathrm{Al}$ is the identification of a suitable reaction coordinate. The size of the largest solid cluster, $n_{s}$, is a first, intuitive choice as it was shown to provide a complete description of the nucleation process in many systems, including LJ, ${ }^{54}$ unary metals, ${ }^{37}$ and soft core colloids. ${ }^{17}$ In Fig. 1(a), the averaged committor projected onto $n_{s}$ from the RPE is presented. The committor increases monotonically with the typical shape, indicating a good correlation between $n_{s}$ and $\bar{p}_{B}$. The averaged committor is, however, not sufficient to evaluate the quality of a CV as a reaction coordinate. Previous studies of crystal nucleation in pure Ni showed that even though the averaged committor exhibits a good correlation with several CVs such as the size of the crystalline core $n_{c}$, this parameter is unable to capture the entire nucleation process, and the corresponding free energy barrier is significantly underestimated.

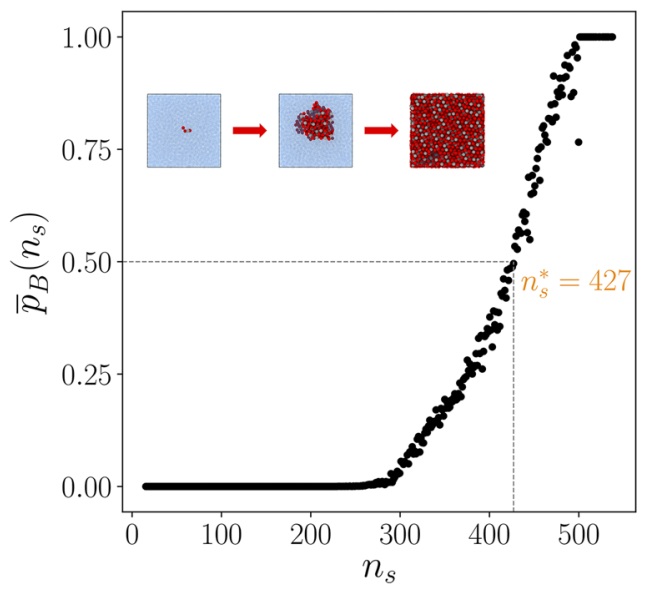

(a)

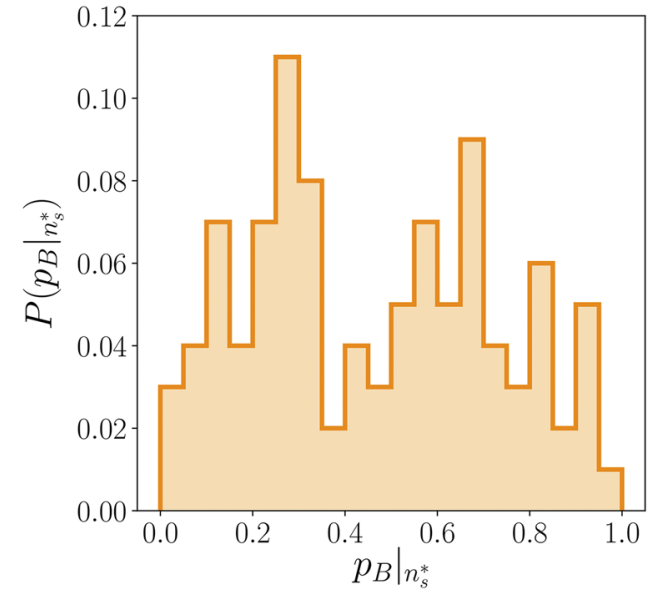

(b)

FIG. 1. (a) Averaged committor $\bar{p}_{B}\left(n_{s}\right)$ projected from the RPE. $\bar{p}_{B}\left(n_{s}\right)$ and $n_{s}$ are closely correlated, and the assumed transition state is at $n_{s}^{*}=427$. The inset illustrates nucleation and crystallization in $\mathrm{Ni}_{3} \mathrm{Al}$. Particles in the liquid phase are shown in transparent blue, $\mathrm{Ni}$ and $\mathrm{Al}$ atoms in the solid phase in red and gray, respectively. (b) Committor distribution $P\left(p_{B} \mid n_{s}^{*}\right)$ extracted from 100 transition state configurations with $n_{s}^{*}=427$. 
To provide a quantitative analysis of the quality of $n_{s}$ as RC, we perform a committor analysis with configurations belonging to the apparent TS $n_{s}^{*}=427$ with $\bar{p}_{B}\left(n_{s}^{*}\right)=0.5$. We collect 100 TS configurations to compute the committor distribution $P\left(\left.p_{B}\right|_{n_{s}^{*}}\right)$ according to Eq. (1). As shown in Fig. 1(b), the committor distribution does not yield a single narrow peak at $p_{B} \approx 0.5$ that is characteristic of the TS ensemble but is spread out over the entire range of committor values. The size of the largest cluster $n_{s}$, therefore, does not yield a good approximation to the RC despite its reasonable correlation with the averaged committor.

To understand why this widely and successfully used CV fails to describe the nucleation mechanism in $\mathrm{Ni}_{3} \mathrm{Al}$, we analyze the structural composition of the growing clusters. Unlike in pure $\mathrm{Ni}$ or $\mathrm{Al}$ where the core of the solid clusters is dominated by $\mathrm{fcc}^{37,38}$ in $\mathrm{Ni}_{3} \mathrm{Al}$, we find a mixture of fcc, bcc, and random stacking of hcp (rhcp). More specifically, two nucleation pathways seem to exist that lead to the formation of fcc and bcc phases, respectively. This can, for example, be seen from the structural composition of configurations at the supposed TS with $n_{s}^{*}=427$. The distribution of the phase fractions of fcc and bcc in the presumed critical clusters in Fig. 2(a) is approximately bimodal: $\frac{n_{s}^{\text {fcc }}}{n_{s}}$ has two peaks around 0.3 and 0.0 , and $\frac{n_{s}^{\text {bcc }}}{n_{s}}$ at 0.6 and 0.0 , respectively. Moreover, the formation of fcc and bcc in the growing clusters appears to be mutually hindered. As shown in Fig. 2(b), the amount of fcc (bcc) substantially increases only in clusters with a rather low bcc (fcc) content. Smaller (pre-critical) and larger (post-critical) clusters exhibit the same accumulation of either fcc or bcc, which indicates that there is a competition between fcc and bcc during nucleation and growth, leading to two separate reaction channels. Similar to the nucleation in unary metals, ${ }^{37}$ we observe the formation of pre-structured liquid ${ }^{18}$ and rhcp for both nucleation pathways.

The structural composition of the small nuclei directly affects the formation of specific polymorphs in the final bulk phase. MD simulations started from presumed TS configurations with clusters that were predominantly composed of fcc or bcc showed that the solidified bulk phase inherits the structure of the initial nuclei. The selection of different polymorphs thus takes place in the early stages of nucleation triggered by the structural composition of the small, initial clusters.

The existence of competing nucleation pathways was also found for the crystallization in LJ systems, ${ }^{6,9}$ methane hydrates, ${ }^{11}$ and mixtures of oppositely charged colloidal particles. ${ }^{28,29}$ During nucleation in a LJ liquid, the critical clusters can be either small, compact, and mostly fcc or large, loosely packed, and more bcclike. ${ }^{6}$ In methane hydrates, two nucleation pathways were identified at moderate undercooling: one toward the thermodynamically stable crystalline hydrate and the other resulting in a metastable amorphous phase. ${ }^{11}$ In the mixture of oppositely charged colloidal particles, competing nucleation pathways were found to coexist in a broad reaction channel with selection occurring near the barrier top, leading to different bulk phases of charge-disordered fcc and $\mathrm{CsCl}$ ordered bcc. ${ }^{29}$ In $\mathrm{Ni}_{3} \mathrm{Al}$, the thermodynamically stable phase is $\mathrm{L}_{2}$ ordered fcc, but there are several chemically ordered and disordered metastable phases including bcc and hcp. ${ }^{31}$ Experimental studies on crystallization in $\mathrm{Ni}-\mathrm{Al}$ alloys indeed revealed a variety of possible transitions between ordered and disordered fcc and bcc phases, depending on the composition and the applied undercooling. ${ }^{34}$ This supports our findings of a competition between the nucleation and growth of either fcc or bcc in $\mathrm{Ni}_{3} \mathrm{Al}$. The relative probability of these two nucleation mechanisms could, however, not be assessed within the current study. Depending on the composition and undercooling, the path probability density in the two reaction channels varies resulting in a weighted contribution of the different paths to the overall nucleation process.

Since the nucleation mechanism strongly depends on the competition between different crystal structures in the growing cluster, it

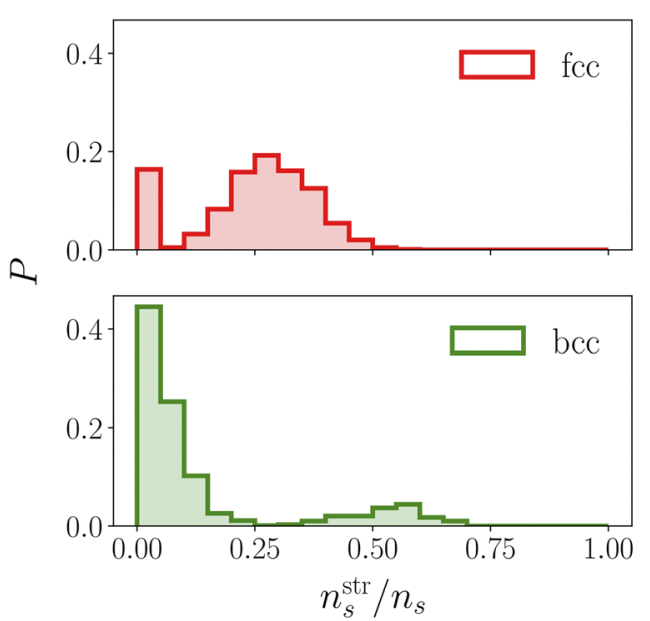

(a)

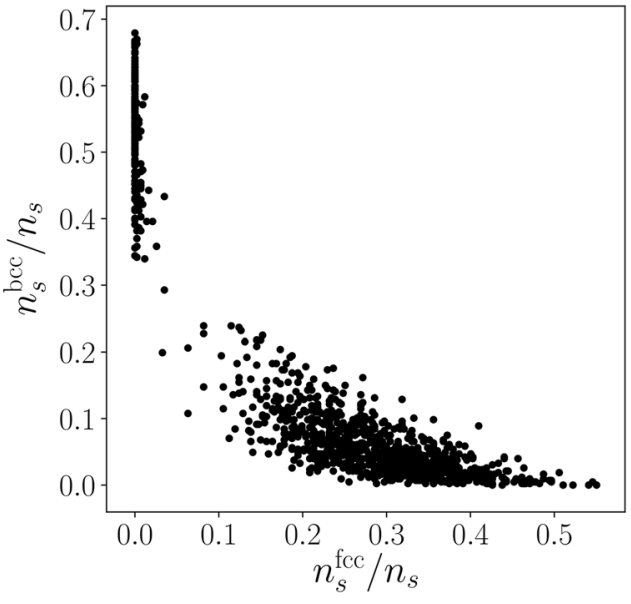

(b)

FIG. 2. (a) Distribution of the phase fractions of fcc (red) and bcc (green) in clusters with a presumed critical size $n_{s}^{*}=427$ computed from 1087 configurations in the path ensemble. (b) Correlation between the phase fractions of fcc and bcc. 
becomes clear why a CV that only measures the size, $n_{s}$, is not sufficient as a reaction coordinate. To obtain a more suitable description, we add in a next step a measure of the crystallinity to the reaction coordinate.

\section{B. Importance of crystallinity}

The global crystallinity $Q_{6}^{\mathrm{cl}}$ (Sec. II C 3) was suggested as an important parameter in the description of nucleation in a LJ liquid where likewise the nucleation mechanism proceeded via several different pathways. ${ }^{6}$ Here, we project the averaged committor from the RPE on both the size of the largest cluster $n_{s}$ and the crystallinity $Q_{6}^{\mathrm{cl}}$, as shown in Fig. 3(a). The TS region with $\bar{p}_{B}\left(n_{s}, Q_{6}^{\mathrm{cl}}\right) \approx 0.5$ exhibits a non-linear dependence on the two CVs, indicating that at least a two-dimensional $\mathrm{RC}$ is required to capture the nucleation mechanism. The presumed TS [red area in Fig. 3(a)] covers a fairly wide range of critical cluster sizes, $300<n_{s}^{*}<460$, and crystallinity values, $0.28<Q_{6}^{\mathrm{cl}^{*}}<0.42$. Critical clusters can be either small with high crystallinity $\left(n_{s} \approx 320, Q_{6}^{\mathrm{cl}} \geq 0.39\right)$, composed of predominantly fcc, negligible bcc, and some rhcp, or they can be large with lower crystallinity $\left(n_{s} \approx 458, Q_{6}^{\mathrm{cl}} \leq 0.33\right)$, consisting of mainly rhcp with comparable amounts of fcc and bcc (see Fig. S2 of the supplementary material). The variety of cluster sizes and crystallinity in the TS ensemble further corroborates the existence of multiple reaction channels and the necessity of a multi-dimensional RC.

To evaluate the quality of our two-dimensional RC model, we performed a committor analysis on the presumed TS ensemble for both small/high-crystallinity and large/low-crystallinity clusters. The committor distribution $P\left(\left.p_{B}\right|_{n_{s}^{*}, Q_{6}^{\mathrm{cl}}}\right)$ computed from 214 configurations with small, compact nuclei satisfying $n_{s}^{*}=320 \pm 5$ and $Q_{6}^{\mathrm{cl}^{*}} \geq 0.39$ is shown in Fig. 3(b). The committor values still extend over the entire range, but there is a slight increase in the probability distribution for low $\left(p_{B} \approx 0.0\right)$ and high $\left(p_{B} \approx 1.0\right)$ values. For large/low-crystallinity clusters of $n_{s}^{*}=458, Q_{6}^{\mathrm{c}^{*}} \leq 0.33$ (54 configurations), the committor distribution $P\left(\left.p_{B}\right|_{n_{s}^{*}, Q_{6}^{\mathrm{c}^{*}}}\right)$ is also spread over the whole range of committor values with a slight increase in the range of $0.2-0.6$ (Fig. S3a of the supplementary material). Even though the two-dimensional RC model somewhat improves the description of the nucleation mechanism and both $n_{s}$ and $Q_{6}^{\mathrm{cl}}$ are important characteristics, there is still an additional component missing.

\section{Chemical short-range order in the growing cluster}

As a measure of how much an additional CV might improve the $\mathrm{RC}$, we analyze their respective correlation with the committor for fixed values of $n_{s}$ and $Q_{6}^{\mathrm{cl}}$. Specifically, we evaluate 20 different parameters that characterize the crystal structure as well as the chemical composition and order as introduced in Sec. II C. For small, compact clusters $\left(n_{s}^{*}=320 \pm 5, Q_{6}^{\mathrm{c}^{*}} \geq 0.39\right)$, all CVs that provide additional information about the structure, that is, the number of core $\left(n_{c}\right)$ and skin $\left(n_{\mathrm{sk}}\right)$ atoms, and the fraction of fcc, bcc, hcp, and undefined in the largest cluster and in the core, do not show any significant correlation with the committor (see Fig. S4 of the supplementary material). This indicates that the global crystallinity already captures the important structural characteristic needed to distinguish different pathways during nucleation and growth in $\mathrm{Ni}_{3} \mathrm{Al}$. Furthermore, the chemical composition does not play any role as an additional parameter in the $\mathrm{RC}$. The amount of $\mathrm{Ni}$ and $\mathrm{Al}$ is approximately constant in the liquid as well as in the solid clusters, also when further separated into core and skin atoms or the different crystalline phases (see Fig. S4 of the supplementary material). Similar uncorrelated behavior of structural CVs and chemical composition with the committor is also observed for large/low-crystallinity clusters $\left(n_{s}^{*}=458, Q_{6}^{\mathrm{c}^{*}} \leq 0.33\right)$.

The chemical short-range order, however, does exhibit a strong correlation with the committor for both the first and second nearest

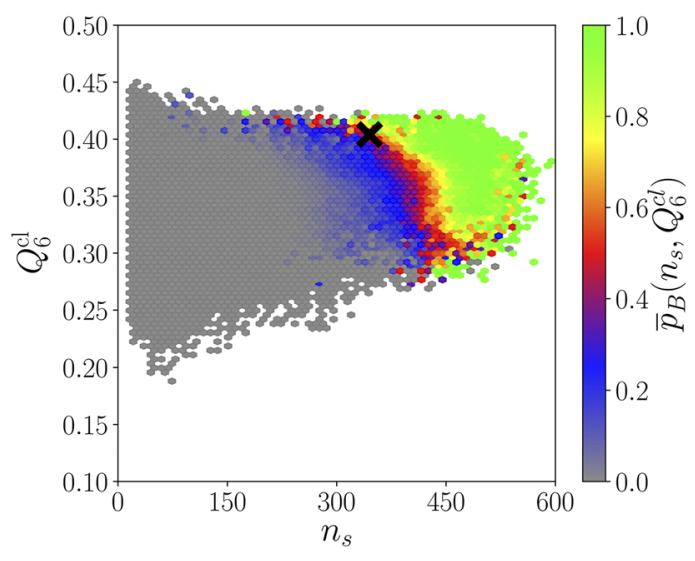

(a)

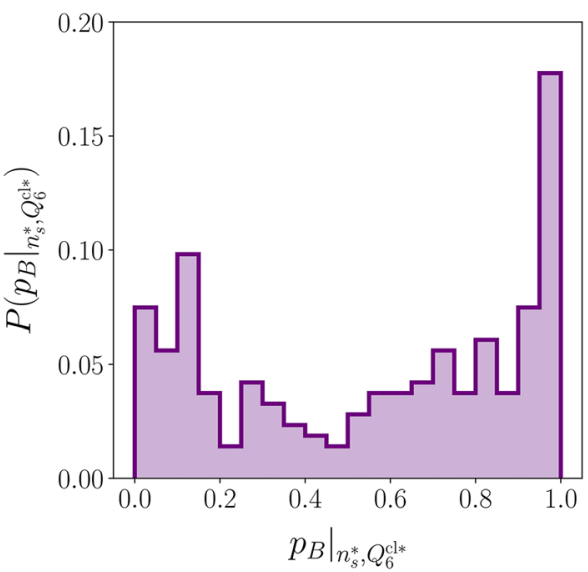

(b)

FIG. 3. (a) Averaged committor $\bar{p}_{B}\left(n_{s}, Q_{6}^{c l}\right)$ projected from the RPE. The TS region $\bar{p}_{B}\left(n_{s}, Q_{6}^{c l}\right) \approx 0.5$ is colored in red. (b) Committor distribution $P\left(\left.p_{B}\right|_{n_{s}^{*}, Q_{6}^{\text {cld }}}\right)$ obtained from 214 (assumed) TS configurations with $n_{s}^{*}=320 \pm 5$ and $Q_{6}^{c^{*}} \geq 0.39$. The TS configurations were chosen from the region of $\bar{p}_{B}\left(n_{s}, Q_{6}^{c l}\right)$ marked by a black cross. 
neighbor shells. In Fig. 4, the committor values of all configurations with small, compact clusters are plotted as a function of $\mathrm{SRO}_{1}$ and $\mathrm{SRO}_{2}$. Clearly, the committor increases with an increase in the SRO toward an $\mathrm{L}_{2}$ ordering with $\mathrm{SRO}_{1}=-1 / 3$ and $\mathrm{SRO}_{2}=1.0$. In these small clusters, the SROs of the first and second nearest neighbors are not independent, confirming the clear correlation between an $\mathrm{L}_{2}$ ordering in the clusters and an increase in $p_{B}$. This suggests that the chemical SRO does indeed provide additional information needed for an appropriate description of the nucleation process. For large clusters with low crystallinity, correlations of $\mathrm{SRO}_{1}$ and $\mathrm{SRO}_{2}$ with the committor are significantly reduced due to the clusters polymorphic composition, where competing rhcp, bcc, and fcc phases make the definition of SRO ambiguous (see Fig. S3b of the supplementary material). In the following, we only focus on the small/high-crystallinity clusters to evaluate the effect of chemical ordering on the RC since the dominant phase fraction of fcc in the core avoids the noise in SRO values that emerges from competing phases.

To confirm our hypothesis that the SRO significantly improves the description of the nucleation mechanism, we perform a committor analysis within the three-dimensional RC space including the size, crystallinity, and short-range order of the largest solid cluster. Specifically, $\mathrm{SRO}_{2}$ is added as a third component to the RC. We define two sets of configurations with small, compact clusters $\left(n_{s}^{*}=320 \pm 5, Q_{6}^{\mathrm{cl}^{*}} \geq 0.39\right)$ : one with high chemical order, $\mathrm{SRO}_{2}$ $\geq 0.75$ (55 configurations), and the other with low chemical order, $\mathrm{SRO}_{2} \leq 0.40$ (54 configurations). The corresponding committor distributions are shown in Fig. 5. The two sets result in clearly separated committor distributions that are sharply peaked at $p_{B} \approx 1.0$ for nuclei with an ordering tendency toward $\mathrm{L}_{2}$ and $p_{B} \approx 0.0$ for clusters showing more chemical disorder. The chemical SRO therefore stabilizes the small, compact clusters, so that they are already

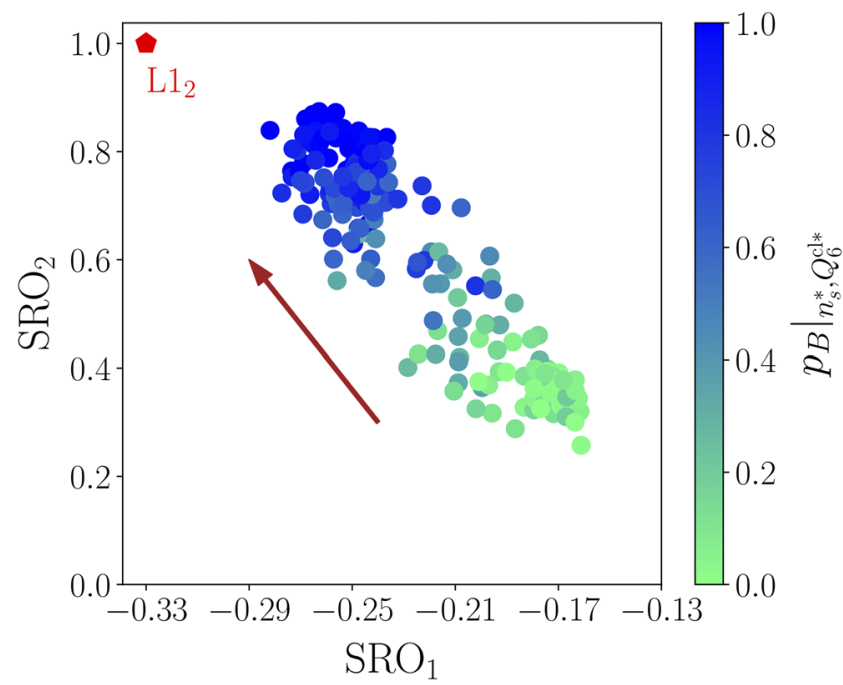

FIG. 4. Committor values of assumed TS configurations for small, compact clusters with $n_{s}^{*}=320 \pm 5$ and $Q_{6}^{c^{*}} \geq 0.39$ as a function of the chemical short-range order. The arrow indicates an increase in SRO values toward $L 1_{2}$ marked by a red pentagon.

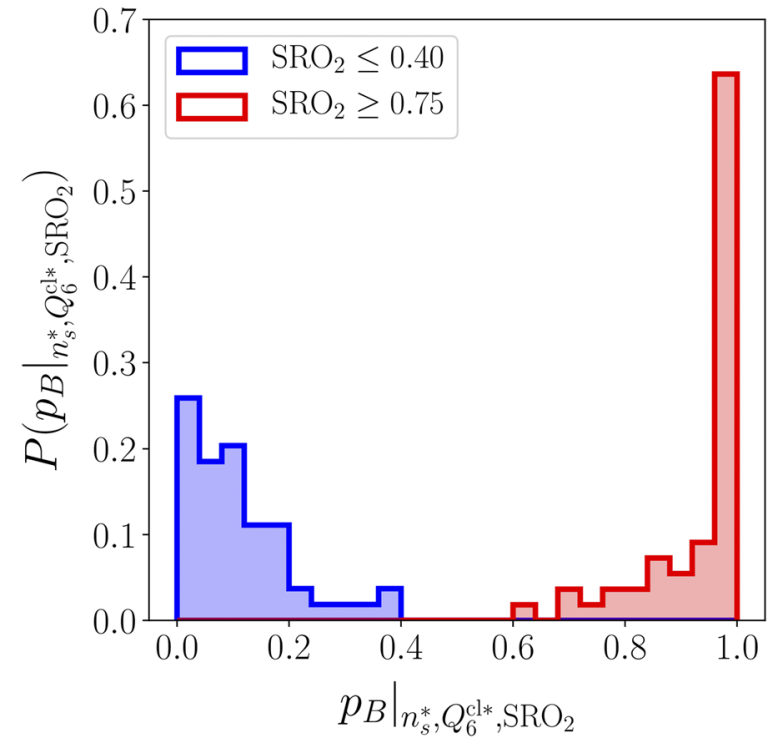

FIG. 5. Committor distributions $P\left(\left.p_{B}\right|_{n_{s}^{*},}, \mathrm{Qdr}_{6}^{\mathrm{dr}}, \mathrm{SRO}_{2}\right)$ for two sets of configurations with the same size $n_{s}^{*}=320 \pm 5$ and crystallinity $Q_{6}^{c^{*}} \geq 0.39$, and low $\mathrm{SRO}_{2} \leq 0.4$ (blue) and high $\mathrm{SRO}_{2} \geq 0.75$ (red), respectively. The distribution for clusters with a chemical ordering close to $L 1_{2}$ is peaked around $p_{B} \approx 1.0$ and for nearly disordered clusters around $p_{B} \approx 0.0$.

beyond the critical nucleus size and continue to grow into the solid bulk phase, whereas the more chemically disordered clusters of the same size and crystallinity dissolve again into the liquid phase. The single peak in both committor distributions suggests that the three-dimensional representation of the $\mathrm{RC}$ considering size, crystallinity, and chemical order can rigorously capture the nucleation mechanism.

\section{Nucleation mechanism and growth in $\mathrm{Ni}_{3} \mathrm{Al}$}

While, in many simple liquids and unary metals, the size of the largest growing cluster is a good approximation to the RC, the nucleation mechanism in $\mathrm{Ni}_{3} \mathrm{Al}$ with several distinct pathways and the formation of various chemically ordered and disordered crystal phases require a multi-dimensional RC that can account for and differentiate between these aspects, as evident from Sec. III C. The importance of the different features and, correspondingly, the probability of different nucleation pathways will also depend on the environmental conditions, such as pressure and undercooling.

In the current study at $20 \%$ undercooling, the chemical SRO plays a key role in stabilizing growing clusters with high crystallinity. This originates in the strong ordering tendency of the bulk phases where for $\mathrm{Ni}_{3} \mathrm{Al}$, the cohesive energy of $\mathrm{L}_{2}\left(E_{\mathrm{Ni}_{3} \mathrm{Al}^{\mathrm{coh}}-\mathrm{Ll}_{2}}^{\mathrm{c}}\right.$ $=-4.63 \mathrm{eV} /$ atom $)$ is about $100 \mathrm{meV} /$ atom lower than the one of disordered fcc $\left(E_{\mathrm{Ni}_{3} \mathrm{Al}-\mathrm{fcc}}^{\mathrm{coh}}=-4.51 \mathrm{eV} /\right.$ atom $)$, calculated using the $\mathrm{Ni}-\mathrm{Al}$ EAM potential. ${ }^{49}$ At finite temperatures, the free energy difference becomes smaller, which is mainly due to configurational entropy, whereas vibrational entropy differences appear to be small between 
the two phases. ${ }^{55}$ Still, experimental and theoretical studies indicate that the ordered $\mathrm{L}_{2}$ phase remains thermodynamically stable up to the melting temperature. ${ }^{56,57}$ Comparing the stability of the small, compact cluster $\left(n_{s}^{*}=320, Q_{6}^{\mathrm{c}^{*}} \geq 0.39\right)$ with high $\left(\mathrm{SRO}_{2}\right.$ $\geq 0.75)$ and low $\left(\mathrm{SRO}_{2} \leq 0.4\right)$ short-range order, we find that the average potential energy of the ordered clusters is $\sim 40 \mathrm{meV} /$ atom lower than that of disordered ones. At smaller undercoolings, we expect the effect to be stronger than at very large undercoolings due to the competition between thermodynamic and kinetic factors. Close to the melting temperature, the nucleation barrier is large, and the corresponding nucleation rate is low. In addition, the diffusion of atoms in the melt is fast. During the formation of solid nuclei, the atoms thus have enough time and are mobile enough to rearrange into the thermodynamically favored phase. At large undercoolings, however, kinetic effects most likely dominate over thermodynamics. The nucleation barrier becomes small, and the formation of solid nuclei occurs rapidly. Together with the slower diffusion of atoms, this leads to the formation of anti-site defects, and the solid phase inherits the chemical order of the liquid instead of relaxing to the thermodynamically stable phase. Consequently, the chemical SRO is not expected to significantly impact the nucleation mechanism at large undercoolings.

Even though we observe a strong effect of the chemical SRO on the stability of the growing clusters in the initial stage of nucleation, the final bulk solids are not $\mathrm{L}_{2}$ ordered in our simulations. To understand this, we have a closer look at the growth stage during solidification. During growth, the formation of a chemically ordered phase is similarly controlled by a competition between diffusion in the melt and the velocity of the solid-liquid interface. To evaluate the ordering and interface velocity during growth, simulations were set up within a supercell containing an $\mathrm{L}_{2}$ ordered bulk phase in contact with liquid $\mathrm{Ni}_{3} \mathrm{Al}$ in the [001] direction (see the supplementary material for further details). After equilibration, the system was kept at $1 \%$ undercooling where the liquid phase is quickly solidified by growth from the solid-liquid interface. Even at this very small undercooling, the estimated interface velocity is high $(v \approx 3 \mathrm{~m} / \mathrm{s})$. As a result, the solidified bulk phase does not grow with $\mathrm{L}_{2}$ ordering but exhibits the same chemical SRO as the liquid phase. This disorder trapping ${ }^{58,59}$ of chemical species is expected whenever the growth velocity exceeds the mobility of atoms in the melt. Experimental studies ${ }^{60}$ of rapid solidification in $\mathrm{Ni}-\mathrm{Al}$ alloys also observed disorder trapping at the crystal growth velocity of $v \approx 4 \mathrm{~m} / \mathrm{s}$ in $\mathrm{Ni}_{3} \mathrm{Al}$. The growth of the ordered $\mathrm{L}_{2}$ phase is predicted to be possible only at the growth velocity well below $1 \mathrm{~m} / \mathrm{s}^{34,61}$ The chemical SRO thus strongly influences the stability of the growing clusters in the initial stage of nucleation, while it only marginally affects the growth stage due to the large solid-liquid interface velocities accompanied by disorder trapping.

\section{CONCLUSION}

In summary, we have identified a multi-dimensional RC as a suitable descriptor for the nucleation process in $\mathrm{Ni}_{3} \mathrm{Al}$ by applying a committor analysis of several CVs on configurations obtained from TIS ensembles. In contrast to unary metals ${ }^{37,38}$ and other bimetallic alloys, ${ }^{19-22}$ the nucleation mechanism in $\mathrm{Ni}_{3} \mathrm{Al}$ exhibits particular complexity that arises from the competition of crystalline structures and chemical ordering. Although the size of the largest solid cluster was found to be strongly correlated with the averaged committor and therefore is a key descriptor of the mechanism, our analysis shows that this order parameter is not sufficient as the RC model of homogeneous nucleation for this bimetallic compound. Indeed, the structural analysis of the nuclei at fixed critical size obtained from the path ensemble revealed the appearance of various crystalline structures strongly indicating the existence of several nucleation channels. Consequently, the RC of the nucleation process in $\mathrm{Ni}_{3} \mathrm{Al}$ is enhanced by a crystallinity parameter $Q_{6}^{\mathrm{cl}}$, similar to what was found by Moroni et al. ${ }^{6}$ for nucleation in a LJ system. Further analysis of the correlation of $\mathrm{CV}$ candidates with the committor unraveled the crucial role of the chemical short-range order to stabilize solid clusters and promote critical fluctuations. For the same size and crystallinity, nuclei with an increase in the chemical order toward the $\mathrm{L}_{2}$ phase tend to grow and solidify, whereas nearly chemically disordered clusters eventually dissolve in the melt. Unlike other bimetallics that form solid solutions, ${ }^{19-22}$ the chemical composition of the clusters does not play a role in the nucleation mechanism of $\mathrm{Ni}_{3} \mathrm{Al}$, as shown by the poor correlation of this $\mathrm{CV}$ with the committor, and thus, its negligible improvement of the RC model. The chemical composition remains essentially constant for the growing nuclei and does not impact the nucleation mechanism, whereas the strong ordering tendency of the chemical elements in $\mathrm{Ni}_{3} \mathrm{Al}$ measured by the SRO parameter strongly enhances the RC.

Our results reveal that a comprehensive description of the nucleation mechanism in $\mathrm{Ni}_{3} \mathrm{Al}$ requires taking into account the interplay between cluster size, crystallinity, and short-range order. In general, we expect that multi-dimensional models of the RC as obtained from our extensive statistical analysis of the committor and path ensemble in $\mathrm{Ni}_{3} \mathrm{Al}$ are of key importance in the characterization of nucleation mechanisms in complex alloys.

\section{SUPPLEMENTARY MATERIAL}

See the supplementary material for additional details on $\mathrm{Ni}_{3} \mathrm{Al} \bar{q}_{4}-\bar{q}_{6}$ map for structure identification, structural compositions of presumed critical clusters, committor distribution of large/low-crystallinity critical clusters, and correlation between chemical short-range order and committor, correlation of various collective variables with the committor for small/high-crystallinity clusters, and rapid growth simulations in $\mathrm{Ni}_{3} \mathrm{Al}$.

\section{ACKNOWLEDGMENTS}

Y.L. acknowledges the Ph.D. fellowship from the International Max Planck Research School for Interface Controlled Materials for Energy Conversion (IMPRS-SurMat). The authors acknowledge financial support from the Deutsche Forschungsgemeinschaft (DFG) through Project Nos. 262052203 and 211503459 (C2 of the collaborative research center SFB/TR 103). The authors acknowledge computing time by the Center for Interface-Dominated High Performance Materials (ZGH, Ruhr-Universität Bochum).

\section{DATA AVAILABILITY}

The data that support the findings of this study are available from the corresponding author upon reasonable request. 


\section{REFERENCES}

${ }^{1}$ G. C. Sosso, J. Chen, S. J. Cox, M. Fitzner, P. Pedevilla, A. Zen, and A. Michaelides, Chem. Rev. 116, 7078 (2016).

${ }^{2}$ J. Russo and H. Tanaka, J. Chem. Phys. 145, 211801 (2016).

${ }^{3}$ R. Becker and W. Döring, Ann. Phys. 416, 719 (1935).

${ }^{4}$ K. Binder, Rep. Prog. Phys. 50, 783 (1987).

${ }^{5}$ A. C. Pan and D. Chandler, J. Phys. Chem. B 108, 19681 (2004).

${ }^{6}$ D. Moroni, P. R. Ten Wolde, and P. G. Bolhuis, Phys. Rev. Lett. 94, 235703 (2005).

${ }^{7}$ F. Trudu, D. Donadio, and M. Parrinello, Phys. Rev. Lett. 97, 105701 (2006).

${ }^{8}$ T. Schilling, H. J. Schöpe, M. Oettel, G. Opletal, and I. Snook, Phys. Rev. Lett. 105, 025701 (2010).

${ }^{9}$ G. T. Beckham and B. Peters, J. Phys. Chem. Lett. 2, 1133 (2011).

${ }^{10}$ W. Lechner, C. Dellago, and P. G. Bolhuis, J. Chem. Phys. 135, 154110 (2011).

${ }^{11}$ T. A. Berendsen, P. G. Bolhuis et al., Proc. Natl. Acad. Sci. U. S. A. 116, 19305 (2019).

${ }^{12}$ P. M. Piaggi, O. Valsson, and M. Parrinello, Phys. Rev. Lett. 119, 015701 (2017).

${ }^{13}$ P. G. Bolhuis, D. Chandler, C. Dellago, and P. L. Geissler, Annu. Rev. Phys. Chem. 53, 291 (2002).

${ }^{14}$ R. Du, V. S. Pande, A. Y. Grosberg, T. Tanaka, and E. S. Shakhnovich, J. Chem. Phys. 108, 334 (1998).

${ }^{15}$ P. L. Geissler, C. Dellago, and D. Chandler, J. Phys. Chem. B 103, 3706 (1999).

${ }^{16}$ B. Peters and B. L. Trout, J. Chem. Phys. 125, 054108 (2006).

${ }^{17}$ W. Lechner, C. Dellago, and P. G. Bolhuis, Phys. Rev. Lett. 106, 085701 (2011).

${ }^{18}$ G. Díaz Leines and J. Rogal, J. Phys. Chem. B 122, 10934 (2018).

${ }^{19} \mathrm{C}$. Desgranges and J. Delhommelle, J. Am. Chem. Soc. 136, 8145 (2014).

${ }^{20}$ C. Desgranges and J. Delhommelle, J. Phys. Chem. C 120, 27657 (2016).

${ }^{21}$ S. Bechelli, B. Gonzalez, V. Piquet, I. Essafri, C. Desgranges, and J. Delhommelle, J. Phys. Chem. B 121, 8558 (2017).

${ }^{22}$ K. D. Watson, S. E. T. Nguelo, C. Desgranges, and J. Delhommelle, CrystEngComm 13, 1132 (2011).

${ }^{23}$ J. Anwar and P. K. Boateng, J. Am. Chem. Soc. 120, 9600 (1998).

${ }^{24}$ T. Gruhn and P. A. Monson, Phys. Rev. E 64, 061703 (2001).

${ }^{25} \mathrm{M}$. Mucha and P. Jungwirth, J. Phys. Chem. B 107, 8271 (2003).

${ }^{26}$ D. Zahn, Phys. Rev. Lett. 92, 040801 (2004).

${ }^{27}$ S. Punnathanam and P. A. Monson, J. Chem. Phys. 125, 024508 (2006).

${ }^{28}$ E. Sanz, C. Valeriani, D. Frenkel, and M. Dijkstra, Phys. Rev. Lett. 99, 055501 (2007).

${ }^{29}$ B. Peters, J. Chem. Phys. 131, 244103 (2009).

${ }^{30}$ D. P. Pope and R. Darolia, MRS Bull. 21, 30 (1996).

${ }^{31}$ J.-h. Xu, B. I. Min, A. J. Freeman, and T. Oguchi, Phys. Rev. B 41, 5010 (1990).
${ }^{32}$ T. B. Massalski, ASM Int. 3, 2874 (1992).

${ }^{33}$ W. Huang and Y. A. Chang, Intermetallics 6, 487 (1998).

${ }^{34}$ H. Assadi, M. Barth, A. L. Greer, and D. M. Herlach, Acta Mater. 46, 491 (1998).

${ }^{35}$ T. S. Van Erp and P. G. Bolhuis, J. Comput. Phys. 205, 157 (2005).

${ }^{36}$ C. Dellago, P. Bolhuis, and P. L. Geissler, Adv. Chem. Phys. 123, 1 (2002).

${ }^{37}$ G. Díaz Leines, R. Drautz, and J. Rogal, J. Chem. Phys. 146, 154702 (2017).

${ }^{38}$ C. Desgranges and J. Delhommelle, J. Am. Chem. Soc. 129, 7012 (2007).

${ }^{39}$ P. G. Bolhuis and W. Lechner, J. Stat. Phys. 145, 841 (2011).

${ }^{40}$ J. Rogal, W. Lechner, J. Juraszek, B. Ensing, and P. G. Bolhuis, J. Chem. Phys. 133, 174109 (2010).

${ }^{41}$ B. Peters, G. T. Beckham, and B. L. Trout, J. Chem. Phys. 127, 034109 (2007).

${ }^{42}$ W. Lechner, J. Rogal, J. Juraszek, B. Ensing, and P. G. Bolhuis, J. Chem. Phys. 133, 174110 (2010).

${ }^{43}$ P. J. Steinhardt, D. R. Nelson, and M. Ronchetti, Phys. Rev. B 28, 784 (1983).

${ }^{44}$ P. R. Ten Wolde, M. J. Ruiz-Montero, and D. Frenkel, Phys. Rev. Lett. 75, 2714 (1995).

${ }^{45}$ J. Bokeloh, R. E. Rozas, J. Horbach, and G. Wilde, Phys. Rev. Lett. 107, 145701 (2011).

${ }^{46}$ W. Lechner and C. Dellago, J. Chem. Phys. 129, 114707 (2008).

${ }^{47}$ S. Jungblut and C. Dellago, Eur. Phys. J. E 39, 77 (2016).

${ }^{48}$ I. S. Atanasov and M. Hou, Eur. Phys. J. D 52, 51 (2009).

${ }^{49}$ G. P. Purja Pun and Y. Mishin, Philos. Mag. 89, 3245 (2009).

${ }^{50}$ S. Plimpton, J. Comput. Phys. 117, 1 (1995).

51 “The TPS-wrapper, a PYTHON-based wrapper to perform transition path sampling coupled with several molecular dynamics drivers, is developed at ICAMS, Ruhr-Universität Bochum. The package can be requested from the corresponding authors via email."

${ }^{52}$ T. S. van Erp, Phys. Rev. Lett. 98, 268301 (2007).

${ }^{53}$ P. G. Bolhuis, J. Chem. Phys. 129, 114108 (2008).

${ }^{54}$ J. A. Van Meel, A. J. Page, R. P. Sear, and D. Frenkel, J. Chem. Phys. 129, 204505 (2008).

${ }^{\mathbf{5 5}}$ A. van de Walle, G. Ceder, and U. V. Waghmare, Phys. Rev. Lett. 80, 4911 (1998).

${ }^{56}$ R. W. Cahn, P. A. Siemers, J. E. Geiger, and P. Bardhan, Acta Metall. 35, 2737 (1987).

${ }^{57}$ M. F. Michelon and A. Antonelli, Phys. Rev. B 81, 094204 (2010).

${ }^{58}$ W. J. Boettinger and M. J. Aziz, Acta Metall. 37, 3379 (1989).

${ }^{59}$ M. J. Aziz and W. J. Boettinger, Acta Metall. Mater. 42, 527 (1994).

${ }^{60}$ M. Barth, B. Wei, D. M. Herlach, and B. Feuerbacher, Mater. Sci. Eng.: A 178, 305 (1994).

${ }^{61}$ H. Assadi and A. L. Greer, ISIJ Int. 35, 574 (1995). 